\title{
Evaluación mediante enumeración de estados de la confiabilidad del Sistema Interconectado del Norte Grande de Chile (SING)
}

\author{
State enumeration approach in reliability assessment \\ of the Chilean far north power system (SING) \\ Víctor Fuentes Morales ${ }^{1} \quad$ Osman Duarte Moya ${ }^{1}$ \\ Recibido 7 de julio de 2009, aceptado 10 de junio de 2011 \\ Received: July 7, 2009 Accepted: June 10, 2011
}

\begin{abstract}
RESUMEN
En este trabajo se presenta una evaluación de la confiabilidad del SING ${ }^{*}$ chileno mediante el método probabilístico analítico enumeración de estados que permite determinar el índice de indisponibilidad del sistema de transmisión y el factor de sobrecosto asociado. Esta evaluación posibilita un análisis comparativo con el índice de indisponibilidad y el factor de sobrecosto asociado que el agente regulador chileno, $\mathrm{CNE}^{* *}$, entrega para este sistema en los informes técnicos de precios de nudo. De esta manera, los resultados propuestos sugieren una solución a las reiteradas observaciones que realizan las empresas eléctricas del SING en los informes técnicos de precios de nudo, con respecto a los criterios utilizados para modelar y determinar la indisponibilidad de transmisión y los sobrecostos asociados. La evaluación de la confiabilidad se realiza abordando al SING en forma compuesta, es decir, considerando tanto las instalaciones de generación como de transmisión en conjunto.

* SING: Sistema Interconectado del Norte Grande. Sistema de transmisión de energía eléctrica de alto voltaje del norte de Chile.

** CNE: Comisión Nacional de Energía. Organismo gubernamental chileno que fiscaliza los sistemas de transmisión de energía eléctrica.
\end{abstract}

Palabras claves: Evaluación, índice de indisponibilidad, confiabilidad, factor de sobrecosto, método probalístico.

\begin{abstract}
This paper presents a reliability evaluation of the Chilean SING* by means of an analytical and probabilistic method for the enumeration of states, which permits to determine the unavailability index and the associated overcharge factor. This evaluation allows a comparative analysis between the unavailability index and the associated overcharge factor, the $C N E^{* *}$, provides in its technical reports of local the nodal price. Hereby, the proposed results suggest a solution for continuous observations made by SING electric companies in the technical reports of nodal price, with respect to the criteria used for modeling and determining the unavailability of transmition and associated overcharge factor. The reliability evaluation is made considering generation and transmission facilities.

* SING: Northern Interconnected System: Transmission system of high voltage electricity in northern Chile.

** CNE: National Energy Commission: Chilean government agency that oversees the transmission systems of electric power.
\end{abstract}

Keywords: Evaluation, unavailability index, reliability, overcharge factor, probabilistic method.

1 Facultad de Ingeniería. Departamento de Ingeniería Eléctrica. Universidad de Antofagasta. Antofagasta, Chile.

E-mail: vfuentes@uantof.cl; osmanduarte@gmail.com 


\section{INTRODUCCIÓN}

En el ámbito mundial, antiguamente quienes se encargaban de entregar y desarrollar el suministro energético eran pocas empresas que se encontraban verticalmente integradas y de propiedad del Estado, y que, junto con este último, formaban la estructura básica de cualquier sistema eléctrico de potencia en ese entonces. El Estado surgía, bajo este escenario, como un actor principal en el sector energético [1].

Hoy en día se han abierto las puertas a la descentralización de funciones y al establecimiento del libre mercado en los sistemas eléctricos de potencia, situación que fue expandiéndose a numerosos países del mundo. Dicha descentralización de funciones y el establecimiento del libre mercado han provocado profundos cambios y la aparición de nuevas estructuras y organizaciones, que, a fin de cuentas, han marcado un nuevo escenario, bajo el cual se deben desarrollar y operar los sistemas eléctricos de potencia [2].

Es así como en este nuevo entorno de los sistemas eléctricos de potencia, aspectos como la confiabilidad, que conlleva la suficiencia, la seguridad y la calidad del suministro, han cobrado real importancia. Estos conceptos están siendo adaptados a las nuevas estructuras liberales y reguladas, y es en esta dirección donde los sistemas eléctricos, a nivel mundial, apuntan sus organizaciones [1].

Para realizar una evaluación de la confiabilidad en un sistema eléctrico de potencia se deben definir, previamente, dos aspectos importantes, estos son: el método a utilizar para desarrollar la evaluación, y segundo, definir el nivel jerárquico que se desea abordar dentro del sistema eléctrico de potencia. Según [3], las metodologías probabilísticas desarrolladas a través de los años se pueden clasificar en dos grandes grupos: las metodologías probabilísticas basadas en la modelación de los estados y las metodologías probabilísticas basadas en la simulación. Según [3], dentro de las metodologías basadas en la modelación de los estados se encuentra el método probabilístico analítico enumeración de estados, el cual también posee un algoritmo de evaluación de la confiabilidad para un sistema eléctrico de potencia que es común en todos los métodos probabilísticos existentes. Recientemente en
Chile, Larraín [6], basado en el método probabilístico analítico enumeración de estados, realizó un estudio del índice de indisponibilidad de transmisión para el Sistema Interconectado Central chileno (SIC), en el cual se procedió a calcular un índice de indisponibilidad para el sistema de transmisión y el sobrecosto asociado a tal índice.

En el presente trabajo se analiza la indisponibilidad del sistema de transmisión del SING a partir de una evaluación de la confiabilidad utilizando una metodología probabilística, de manera de determinar el índice de indisponibilidad representativo para el sistema de transmisión del SING. Esta evaluación de la confiabilidad se aplica desde la perspectiva de la suficiencia o capacidad, de manera de cuantificar si las instalaciones existentes de transmisión son capaces de abastecer toda la demanda cuando ocurren fallas intempestivas en el sistema. Es importante mencionar que la evaluación se realiza abordando al SING en un nivel jerárquico II, es decir, se modela considerando las instalaciones de generación y transmisión en forma conjunta. Los índices de confiabilidad que se obtienen de esta evaluación son la probabilidad de pérdida de carga, LOLP, en por unidad, la pérdida de carga esperada, LOLE, en horas al año, la potencia esperada no suministrada, PENS, en MW, y la energía esperada no suministrada, EENS, en MWh al año.

En el contexto señalado, este trabajo tiene como objetivo principal analizar y evaluar la confiabilidad del SING mediante el método probabilístico analítico enumeración de estados. Esto permite obtener el índice de indisponibilidad del sistema de transmisión y el factor de sobrecosto asociado, considerando un análisis estático de la operación y con estadísticas de falla estándares para los componentes del sistema.

En la siguiente sección se expone la formulación de la metodología probabilística analítica enumeración de estados, describiendo el algoritmo conceptual común para los métodos probabilísticos que permiten evaluar la confiabilidad de los sistemas eléctricos de potencia. A continuación se aplica la metodología al sistema eléctrico chileno, SING, obteniendo los índices de confiabilidad requeridos y descritos en párrafo anterior. En la siguiente sección se presenta el cálculo del factor de sobrecosto asociado al índice de indisponibilidad del sistema de transmisión, y 
finalmente, se entrega un análisis de los resultados, las conclusiones de la aplicación y los trabajos futuros asociados.

\section{METODOLOGÍA PARA EVALUAR LA CONFIABILIDAD DE UN SISTEMA ELÉCTRICO DE POTENCIA}

La metodología probabilística analítica enumeración de estados busca obtener en forma rápida y concreta los índices de confiabilidad de un sistema eléctrico de potencia. Esta metodología se basa en el estudio de todos los estados posibles que puede presentar un sistema, incorporando, eso sí, algunas simplificaciones inherentes al nivel de complejidad del estudio, dado que un sistema puede ser lo suficientemente complejo como para abordar todos los estados posibles. Es útil analizar los estados en orden creciente de contingencias (caso base sin fallas, luego el criterio $\mathrm{N}-1, \mathrm{~N}-2$, etc.) hasta que la probabilidad asociada a los resultados sea lo suficientemente pequeña como para ser ignorados [5]. En cuanto a las simplificaciones inherentes que se pueden incorporar en la selección de los estados, es práctico analizar sólo los eventos o estados más creíbles. Se puede entender por creíbles aquellos estados que consideran contingencias hasta un nivel determinado, nivel que puede ser, por ejemplo, el número de contingencias simultáneas que puedan ocurrir. Si se opta por realizar esta simplificación, el número de estados analizados en definitiva es un porcentaje pequeño con respecto al total de estados que puede tener el sistema, pero, por el contrario, la suma de las probabilidades de ocurrencia que cada estado seleccionado aporta corresponde a un porcentaje alto, muy cercano al $100 \%$ [6].

\section{Algoritmo conceptual}

Las etapas que forman el algoritmo de evaluación de la confiabilidad en los sistemas eléctricos de potencia, son las siguientes [7]:

- Selección de un estado $\mathbf{x}$ del sistema dentro de un conjunto de estados $\mathbf{X}$ (espacio de todos los estados). En esta etapa se define el escenario a evaluar en términos de disponibilidad de equipos, instalaciones y la carga a abastecer.

- Cálculo de la función de prueba $\mathbf{F}(\mathbf{x})$ para el estado seleccionado. Mediante la función de prueba, es posible evaluar el comportamiento del sistema, analizando bajo condiciones y restricciones impuestas, el abastecimiento o desprendimiento de la carga conectada.

- Cálculo de los índices de confiabilidad mediante el cálculo de la esperanza de la función de prueba, $\mathbf{E}(\mathbf{F}(\mathbf{x}))$.

- Verificación de las condiciones de término. En caso de no cumplirse éstas, continuar con la evaluación.

En el caso del método analítico enumeración de estados, también es posible establecer el procedimiento básico para evaluar la confiabilidad de un sistema eléctrico de potencia.

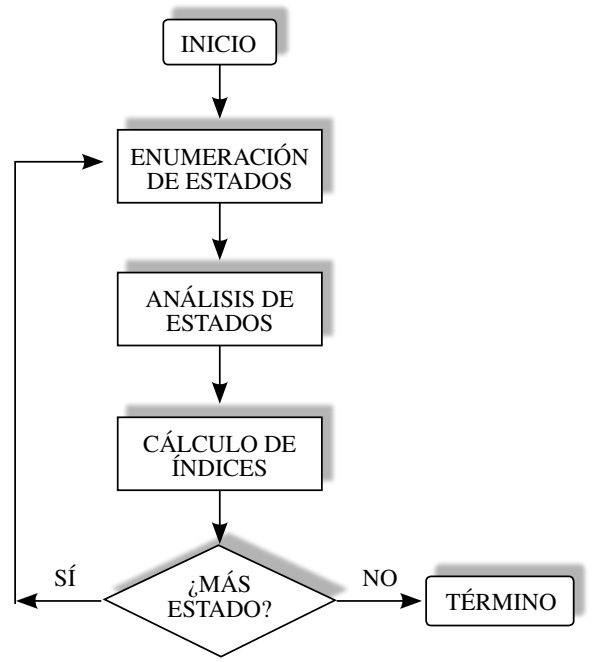

Figura 1. Procedimiento básico para desarrollar la metodología analítica enumeración de estados.

En la Figura 1 se muestran las etapas que componen el procedimiento para evaluar la confiabilidad de un sistema eléctrico de potencia a través del método enumeración de estados. Para seleccionar los estados a evaluar es conveniente utilizar las mejoras que se le han incorporado a dicho método, y que consisten, básicamente, en seleccionar tan solo un subconjunto del conjunto total de estados posibles, en base a criterios tales como el análisis de tan solo los estados más probables o creíbles. Un criterio para seleccionar los estados de falla, tal como lo señala en su tesis de título Larraín [6], es el siguiente:

- En generación, se consideran contingencias de hasta segundo orden, es decir, la salida de 
servicio o falla simultánea de hasta dos unidades generadoras.

- En transmisión, se consideran contingencias de primer orden, ya que la probabilidad de ocurrencia de las contingencias de segundo orden es muy cercana a cero, pero, de todas maneras, no es excluyente incorporar contingencias de mayor orden.

Para calcular la función de prueba del método, ésta dependerá, exclusivamente, del índice de confiabilidad que se desee calcular. Por ejemplo, para calcular el índice probabilidad de pérdida de carga, LOLP, la función de prueba es la siguiente [6]:

$$
F_{1}(x)= \begin{cases}1 & \text { si } x \text { es un estado con falla. } \\ 0 & \text { si } x \text { es un estado sano. }\end{cases}
$$

Para calcular la potencia esperada no suministrada, PENS, la función de prueba a utilizar es la siguiente [6]:

$$
F_{2}(x)= \begin{cases}\varphi & \text { si } x \text { es un estado con falla. } \\ 0 & \text { si } x \text { es un estado sano. }\end{cases}
$$

Donde:

$\varphi$ : Es la potencia no suministrada en MW, para el estado i del sistema.

En general, para obtener los índices de confiabilidad para cada estado seleccionado una vez evaluadas las funciones de prueba, se deben utilizar la siguientes ecuaciones [6]:

$$
\begin{gathered}
\operatorname{LOLP}_{\mathrm{x}}=\mathrm{F}_{1}(\mathrm{x}) \cdot \mathrm{p}_{\mathrm{Si}} \\
\mathrm{PENS}_{\mathrm{x}}=\mathrm{F}_{2}(\mathrm{x}) \cdot \mathrm{p}_{\mathrm{Si}}[\mathrm{MW}] \\
\operatorname{LOLE}_{\mathrm{x}}=\mathrm{LOLP}_{\mathrm{x}} \cdot 8.760[\text { horas } / \text { año }] \\
\mathrm{EENS}_{\mathrm{x}}=\mathrm{EPNS}_{\mathrm{x}} \cdot 8.760 \cdot \mathrm{F}_{\mathrm{C}}[\mathrm{MWh} / \text { año }]
\end{gathered}
$$

Donde:

$\mathrm{p}_{\mathrm{si}}$ : Es la probabilidad de ocurrencia del estado i. $\mathrm{F}_{\mathrm{C}}$ : Es el factor de carga del SING, y 8.760 corresponde al período de estudio que son las horas en un año.
Para obtener, finalmente, los índices globales del sistema, se debe realizar la sumatoria de los índices de cada uno de los estados seleccionados, cuyas ecuaciones son [6]:

$$
\begin{gathered}
\text { LOLP }=\sum_{\mathrm{x}=1}^{\mathrm{n}} \operatorname{LOLP}_{\mathrm{x}} \\
\text { PENS }=\sum_{\mathrm{x}=1}^{\mathrm{n}} \operatorname{PENS}_{\mathrm{x}}[\mathrm{MW}] \\
\mathrm{LOLE}=\sum_{\mathrm{x}=1}^{\mathrm{n}} \operatorname{LOLE}_{\mathrm{x}}[\text { horas/año }] \\
\text { EENS }=\sum_{\mathrm{x}=1}^{\mathrm{n}} \operatorname{EENS}_{\mathrm{x}}[\mathrm{MWh} / \mathrm{año}]
\end{gathered}
$$

Cabe mencionar que los índices definidos mediante las ecuaciones (7), (8), (9) y (10) se deben obtener para el sistema de generación, para el sistema de transmisión y para el sistema compuesto.

\section{Modelo de los componentes para las evaluaciones de confiabilidad}

Un componente del sistema eléctrico se puede modelar por dos estados posibles: disponible e indisponible [5]. El elemento pasa al estado indisponible tras sufrir una falla, volviendo a estar disponible tras su reparación. La transición de estados se representa mediante un modelo de Markov, siendo la tasa de falla, $\lambda$, y la tasa de reparación, $\mu$, los parámetros de falla más importantes para modelar un componente.

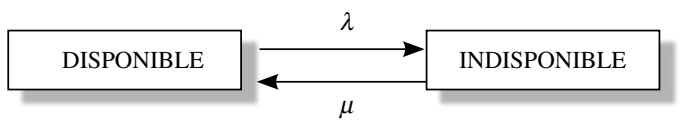

Figura 2. Modelo de Markov para un componente.

La Figura 2 muestra la transición que puede tener un componente cualquiera del sistema durante su vida útil, por lo que conociendo las tasas de falla y reparación del componente la probabilidad de que éste se encuentre en cualquiera de los dos estados posibles se obtiene calcular a través de las siguientes ecuaciones [5]:

$$
\mathrm{p}_{\mathrm{d}}=\frac{\mu}{\lambda+\mu}
$$




$$
\begin{aligned}
& \mathrm{p}_{\mathrm{i}}=\frac{\lambda}{\lambda+\mu} \\
& \mathrm{p}_{\mathrm{d}}+\mathrm{p}_{\mathrm{i}}=1
\end{aligned}
$$

La ecuación (11) permite calcular la probabilidad de encontrar el componente en el estado disponible, que es proporcional a la tasa de reparación $\mu$, e inversamente proporcional a la suma de ambas tasas. Por su parte, la ecuación (12) permite calcular la probabilidad de encontrar al componente en el estado indisponible, que es proporcional a la tasa de falla $\lambda$, e inversamente proporcional a la suma de ambas tasas [8], y finalmente, la ecuación (13) entrega la suma de ambas probabilidades, que debe ser igual a 1.

Los conceptos básicos que se utilizan para la evaluación de la confiabilidad en un sistema compuesto, generación-transmisión, son, principalmente, dos [6]:

- La independencia de los componentes.

- La coherencia del sistema.

La independencia de los componentes es la suposición más fuerte que se adopta en la evaluación de la confiabilidad de sistemas compuestos, ya que implica que los procesos de falla y reparación de los diferentes componentes son, estadísticamente, independientes, es decir, la ocurrencia o no ocurrencia de una condición de falla en algún componente no modifica las probabilidades de falla de los componentes restantes. Por su parte, la coherencia del sistema indica que el rendimiento del sistema no puede ser mejor si un componente que está funcionando falla, y no puede ser peor si un componente que ha fallado es reparado, es decir, si un estado del sistema compuesto contiene componentes que en forma separada producían fallas, entendiéndose por separada a los sistemas de generación y transmisión por sí solos, en el sistema compuesto también producirán una falla.

Un sistema eléctrico, por su parte, está constituido por múltiples componentes: generadores, líneas de transmisión y transformadores, principalmente. En consecuencia, el estado del sistema eléctrico estará definido por el estado de cada componente y la probabilidad de que el sistema se encuentre en dicho estado se obtiene como el producto de las probabilidades individuales, siempre bajo la suposición de que los fallos son, estadísticamente, independientes [5].

Para calcular la probabilidad de ocurrencia de un estado en un sistema con $\mathbf{n}$ componentes, y donde cada componente está modelado por dos estados posibles, se utiliza la siguiente ecuación, asumiendo la independencia de los componentes [6]:

$$
\mathrm{p}_{\mathrm{si}}=\prod_{\mathrm{k} \in \mathrm{U}} \mathrm{p}_{\mathrm{k}} \cdot \prod_{\mathrm{m} \in \mathrm{D}} \mathrm{q}_{\mathrm{m}}
$$

Donde:

$\mathrm{p}_{\mathrm{si}}$ : Es la probabilidad de ocurrencia del estado i.

$\mathrm{U}$ : Es el conjunto de todos los componentes disponibles, en el estado i.

D: Es el conjunto de todos los componentes indisponibles, en el estado i.

$\mathrm{P}_{\mathrm{k}}$ : Es la disponibilidad del componente $\mathrm{k}$, en por unidad.

$\mathrm{q}_{\mathrm{m}}$ : Es la indisponibilidad del componente $\mathrm{m}$, en por unidad.

La ecuación (14) indica que las probabilidades de ocurrencia de un estado en el sistema se obtienen, simplemente, multiplicando las disponibilidades por las indisponibilidades de cada componente, dependiendo si los componentes en el estado i se encuentran en o fuera de servicio.

\section{Análisis de los estados de un sistema}

Es importante tener una comprensión clara de qué ocasiona un problema en el sistema y qué es un problema en una evaluación de la confiabilidad. Generalmente, un sistema eléctrico de potencia se considera en falla si el servicio a las barras de carga se interrumpe o la calidad de abastecimiento llega a ser inaceptable. Tal condición proviene si cualquiera de los siguientes eventos ocurre:

- No hay suficiente generación de energía eléctrica para abastecer toda la demanda del sistema.

- La continuidad del abastecimiento a las barras de carga se interrumpe.

- Las líneas de transmisión de energía eléctrica están sobrecargadas, y

- Las tensiones de barra se encuentran fuera del rango permitido. 
Un problema del sistema debido a cualquiera de las condiciones mencionadas en el párrafo anterior no significa, generalmente, un colapso generalizado del sistema eléctrico de potencia. Si ocurriese, sería por una condición de sobrecarga que se desarrolla en una secuencia de eventos en cascada, terminando en un colapso generalizado del sistema. Esta situación cae en el dominio de la evaluación de la seguridad. En la mayoría de los casos las fallas del sistema son corregidas por acciones correctivas, tales como conmutadores, redespacho de generación o desprendimientos de carga. Los estados de falla que ocasionan desprendimientos de carga y violación de tensiones en las barras contribuyen a los índices de capacidad o suficiencia del sistema. Además, para este trabajo en particular se considerarán parte de los índices de suficiencia los colapsos generalizados del sistema o blackout.

\section{Índices desagregados de un sistema}

Los índices de indisponibilidad representativos que se buscan para la generación y la transmisión del sistema eléctrico de potencia en estudio se calculan a través de los índices desagregados del sistema, en palabras simples, se debe repartir el índice compuesto en los dos sistemas, generación y transmisión. Esta desagregación se calcula mediante las ecuaciones (15), (16), (17) y (18) obtenidas de [6], donde, $\overline{\operatorname{LOLP}}_{\mathrm{G}, \mathrm{T}}$ es el índice desagregado probabilidad de pérdida de carga de generación o de transmisión, $\overline{\mathrm{LOLE}}_{\mathrm{G}, \mathrm{T}}$ es el índice desagregado pérdida de carga esperada de generación o de transmisión, $\overline{\mathrm{PENS}}_{\mathrm{G}, \mathrm{T}}$ es el índice desagregado potencia esperada no suministrada de generación o de transmisión, y finalmente, $\overline{\mathrm{EENS}}_{\mathrm{G}, \mathrm{T}}$ es el índice desagregado energía esperada no suministrada de generación o de transmisión.

$$
\begin{aligned}
& \overline{\operatorname{LOLP}}_{\mathrm{G}, \mathrm{T}}=\frac{\mathrm{LOLP}_{\mathrm{G}, \mathrm{T}}}{\mathrm{LOLP}_{\mathrm{G}}+\mathrm{LOLP}_{\mathrm{T}}} \cdot \mathrm{LOLP}_{\mathrm{C}} \\
& \overline{\mathrm{LOLE}}_{\mathrm{G}, \mathrm{T}}=\frac{\mathrm{LOLE}_{\mathrm{G}, \mathrm{T}}}{\mathrm{LOLE}_{\mathrm{G}}+\mathrm{LOLE}_{\mathrm{T}}} \cdot \mathrm{LOLE}_{\mathrm{C}} \\
& \overline{\mathrm{PENS}}_{\mathrm{G}, \mathrm{T}}=\frac{\mathrm{PENS}_{\mathrm{G}, \mathrm{T}}}{\mathrm{PENS}_{\mathrm{G}}+\mathrm{PENS}_{\mathrm{T}}} \cdot \mathrm{PENS}_{\mathrm{C}} \\
& \overline{\mathrm{EENS}}_{\mathrm{G}, \mathrm{T}}=\frac{\mathrm{EENS}_{\mathrm{G}, \mathrm{T}}}{\mathrm{EENS}_{\mathrm{G}}+\mathrm{EENS}_{\mathrm{T}}} \cdot \mathrm{EENS}_{\mathrm{C}}
\end{aligned}
$$

Donde:

LOLP $_{C}$ : Probabilidad de pérdida de carga del sistema compuesto.

LOLE $_{C}$ : Pérdida de carga esperada del sistema compuesto.

PENS $_{\mathrm{C}}$ : Potencia esperada no suministrada del sistema compuesto.

EENS $_{C}$ : Energía esperada no suministrada del sistema compuesto.

Como nomenclatura en las ecuaciones (15), (16), (17) y (18), los índices desagregados se escriben con una barra sobre su nombre para diferenciarlos de los obtenidos en los sistemas de generación, transmisión y compuesto.

\section{ESTUDIO DE APLICACIÓN}

\section{Consideraciones generales}

Para realizar la evaluación de la confiabilidad del SING se ha representado, a este último, a través de un modelo simplificado similar al propuesto en [9]. El diagrama unilineal resultante consta de 51 barras, 80 tramos de líneas y 10 centrales generadoras. En la Figura 4 se muestra el diagrama simplificado del SING utilizado para modelar su operación.

Con respecto a la demanda del sistema, se considera una demanda anual entre los meses de octubre de 2006 y septiembre de 2007, con datos de periodicidad semanal. La gráfica de la curva de demanda del período especificado se muestra en la Figura 3. De esta demanda surgen los dos niveles de carga, máximo y mínimo, y para ambos niveles de carga se evaluará la confiabilidad del sistema.

La Figura 3 fue confeccionada con datos de demanda cuya periodicidad es semanal, modelando 4 semanas en los 12 meses de un año comprendido entre octubre de 2006 y septiembre de 2007.

La capacidad de generación del SING es representada mediante 17 unidades generadoras, las que corresponden a 10 centrales aproximadamente. La indisponibilidad de las unidades generadoras es modelada usando tasas de falla estándares provenientes de [10]. Por su parte, la capacidad de generación disponible durante el análisis corresponde a un despacho típico en condiciones actuales de disponibilidad de gas, donde la mayor contribución al despacho la realizan unidades a base de carbón y 


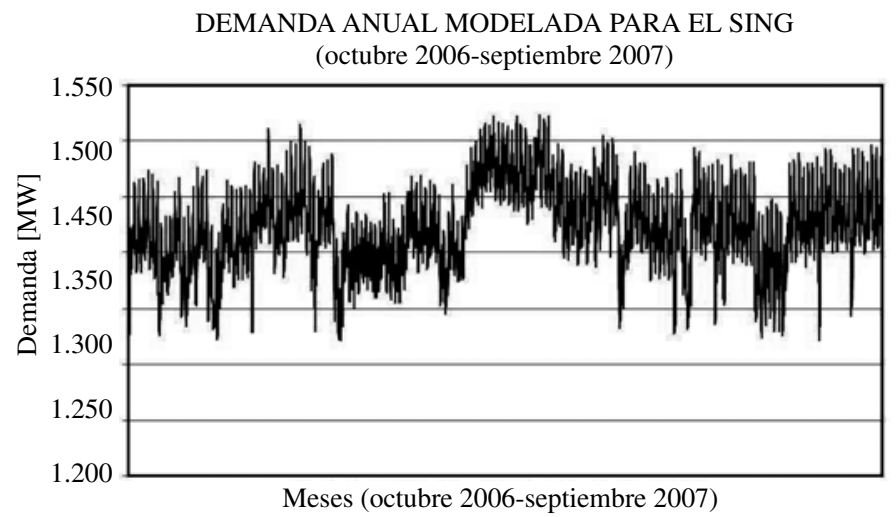

Figura 3. Gráfico de la curva anual de demanda considerada para el SING.

petróleo, y no tanto así, a base a gas. Cabe agregar, además, que las unidades consideradas para el desarrollo del método dependen de su condición de operación según el despacho utilizado. Para evaluar la confiabilidad del SING se analizan tres escenarios independientes, estos son: el sistema de generación considerando $100 \%$ de disponibilidad del sistema de transmisión, el sistema de transmisión considerando $100 \%$ de disponibilidad del sistema de generación y el sistema compuesto generación-transmisión. En estos tres escenarios se obtienen los índices de confiabilidad definidos, para posteriormente obtener los índices desagregados del sistema.

\section{Evaluación de la confiabilidad del SING. Selección de los estados}

El proceso de selección de estados se llevó a cabo en los tres escenarios de análisis definidos. A modo de hacer más explicativo el proceso de selección de los estados, se ejemplificará el proceso de selección de estados con el sistema de generación, donde según la Tabla 2 muestra que los estados seleccionados fueron 137. Los 137 estados surgen de considerar contingencias de hasta segundo orden, es decir, se modela el sistema con la salida de servicio de a lo más dos unidades generadoras en forma simultánea. Luego, del parque generador considerado para el SING de 17 unidades, el despacho típico utilizado indica que sólo 10 unidades se encuentran en servicio, por lo tanto, los 137 estados surgen de la combinación de estas. La Tabla 1 muestra los 137 estados seleccionados y las combinaciones de unidades generadoras fuera de servicio para contingencias de primer y segundo orden.
Tabla 1. Estados seleccionados para el sistema de generación.

\begin{tabular}{|c|c|c|}
\hline Estado & $\begin{array}{c}\text { Unidad fuera } \\
\text { de servicio }\end{array}$ & $\begin{array}{c}\text { Unidad fuera } \\
\text { de servicio }\end{array}$ \\
\hline 1 & 0 & 0 \\
\hline 2 & 0 & CAVA \\
\hline 3 & 0 & CHAP1 \\
\hline 4 & 0 & CHAP2 \\
\hline 5 & 0 & TG12 \\
\hline 6 & 0 & NT02 \\
\hline 7 & 0 & NT01 \\
\hline 8 & 0 & U15 \\
\hline 9 & 0 & CTM1 \\
\hline 10 & 0 & U14 \\
\hline 11 & 0 & CTTAR \\
\hline 12 & 0 & U12 \\
\hline 13 & 0 & U13 \\
\hline 14 & 0 & U10 \\
\hline 15 & 0 & U11 \\
\hline 16 & 0 & MSIQ \\
\hline 17 & 0 & TG2A+0,5TV2C (Diesel) \\
\hline 18 & CAVA & CHAP1 \\
\hline 19 & CAVA & CHAP2 \\
\hline 20 & CAVA & TG12 \\
\hline 21 & CAVA & NT02 \\
\hline 22 & CAVA & NT01 \\
\hline 23 & CAVA & U15 \\
\hline 24 & CAVA & CTM1 \\
\hline 25 & CAVA & U14 \\
\hline 26 & CAVA & CTTAR \\
\hline 27 & CAVA & U12 \\
\hline 28 & CAVA & U13 \\
\hline 29 & CAVA & U10 \\
\hline 30 & CAVA & U11 \\
\hline 31 & CAVA & MSIQ \\
\hline 32 & CAVA & TG2A+0,5TV2C (Diesel) \\
\hline 33 & CHAP1 & CHAP2 \\
\hline
\end{tabular}




\begin{tabular}{|c|c|c|}
\hline Estado & $\begin{array}{c}\text { Unidad fuera } \\
\text { de servicio }\end{array}$ & $\begin{array}{c}\text { Unidad fuera } \\
\text { de servicio }\end{array}$ \\
\hline 34 & CHAP1 & TG12 \\
\hline 35 & CHAP1 & NT02 \\
\hline 36 & CHAP1 & NT01 \\
\hline 37 & CHAP1 & U15 \\
\hline 38 & CHAP1 & CTM1 \\
\hline 39 & CHAP1 & U14 \\
\hline 40 & CHAP1 & CTTAR \\
\hline 41 & CHAP1 & U12 \\
\hline 42 & CHAP1 & U13 \\
\hline 43 & CHAP1 & U10 \\
\hline 44 & CHAP1 & U11 \\
\hline 45 & CHAP1 & MSIQ \\
\hline 46 & CHAP1 & TG2A+0,5TV2C (Diesel) \\
\hline 47 & CHAP2 & TG12 \\
\hline 48 & CHAP2 & NT02 \\
\hline 49 & CHAP2 & NT01 \\
\hline 50 & CHAP2 & U15 \\
\hline 51 & CHAP2 & CTM1 \\
\hline 52 & CHAP2 & U14 \\
\hline 53 & CHAP2 & CTTAR \\
\hline 54 & CHAP2 & U12 \\
\hline 55 & CHAP2 & U13 \\
\hline 56 & CHAP2 & $\mathrm{U} 10$ \\
\hline 57 & CHAP2 & U11 \\
\hline 58 & CHAP2 & MSIQ \\
\hline 59 & CHAP2 & TG2A+0,5TV2C (Diesel) \\
\hline 60 & TG12 & NT02 \\
\hline 61 & TG12 & NT01 \\
\hline 62 & TG12 & U15 \\
\hline 63 & TG12 & CTM1 \\
\hline 64 & TG12 & U14 \\
\hline 65 & TG12 & CTTAR \\
\hline 66 & TG12 & U12 \\
\hline 67 & TG12 & U13 \\
\hline 68 & TG12 & U10 \\
\hline 69 & TG12 & U11 \\
\hline 70 & TG12 & MSIQ \\
\hline 71 & TG12 & TG2A+0,5TV2C (Diesel) \\
\hline 72 & NT02 & NT01 \\
\hline 73 & NT02 & U15 \\
\hline 74 & NT02 & CTM1 \\
\hline 75 & NT02 & U14 \\
\hline 76 & NT02 & CTTAR \\
\hline 77 & NT02 & U12 \\
\hline 78 & NT02 & U13 \\
\hline 79 & NT02 & U10 \\
\hline 80 & NT02 & U11 \\
\hline 81 & NT02 & MSIQ \\
\hline 82 & NT02 & TG2A+0,5TV2C (Diesel) \\
\hline 83 & NT01 & U15 \\
\hline 84 & NT01 & CTM1 \\
\hline 85 & NT01 & U14 \\
\hline
\end{tabular}

\begin{tabular}{|c|c|c|}
\hline Estado & $\begin{array}{c}\text { Unidad fuera } \\
\text { de servicio }\end{array}$ & $\begin{array}{c}\text { Unidad fuera } \\
\text { de servicio }\end{array}$ \\
\hline 86 & NT01 & CTTAR \\
\hline 87 & NT01 & U12 \\
\hline 88 & NT01 & U13 \\
\hline 89 & NT01 & U10 \\
\hline 90 & NT01 & U11 \\
\hline 91 & NT01 & MSIQ \\
\hline 92 & NT01 & TG2A+0,5TV2C (Diesel) \\
\hline 93 & U15 & CTM1 \\
\hline 94 & U15 & U14 \\
\hline 95 & $\mathrm{U} 15$ & CTTAR \\
\hline 96 & U15 & $\mathrm{U} 12$ \\
\hline 97 & U15 & U13 \\
\hline 98 & U15 & U10 \\
\hline 99 & U15 & U11 \\
\hline 100 & U15 & MSIQ \\
\hline 101 & U15 & TG2A+0,5TV2C (Diesel) \\
\hline 102 & CTM1 & U14 \\
\hline 103 & CTM1 & CTTAR \\
\hline 104 & CTM1 & U12 \\
\hline 105 & CTM1 & U13 \\
\hline 106 & CTM1 & U10 \\
\hline 107 & CTM1 & U11 \\
\hline 108 & CTM1 & MSIQ \\
\hline 109 & CTM1 & TG2A+0,5TV2C (Diesel) \\
\hline 110 & U14 & CTTAR \\
\hline 111 & U14 & $\mathrm{U} 12$ \\
\hline 112 & U14 & U13 \\
\hline 113 & U14 & $\mathrm{U} 10$ \\
\hline 114 & U14 & U11 \\
\hline 115 & U14 & MSIQ \\
\hline 116 & U14 & TG2A+0,5TV2C (Diesel) \\
\hline 117 & CTTAR & $\mathrm{U} 12$ \\
\hline 118 & CTTAR & U13 \\
\hline 119 & CTTAR & U10 \\
\hline 120 & CTTAR & U11 \\
\hline 121 & CTTAR & MSIQ \\
\hline 122 & CTTAR & TG2A+0,5TV2C (Diesel) \\
\hline 123 & $\mathrm{U} 12$ & U13 \\
\hline 124 & $\mathrm{U} 12$ & $\mathrm{U} 10$ \\
\hline 125 & $\mathrm{U} 12$ & U11 \\
\hline 126 & $\mathrm{U} 12$ & MSIQ \\
\hline 127 & $\mathrm{U} 12$ & TG2A+0,5TV2C (Diesel) \\
\hline 128 & U13 & U10 \\
\hline 129 & U13 & U11 \\
\hline 130 & U13 & MSIQ \\
\hline 131 & U13 & TG2A+0,5TV2C (Diesel) \\
\hline 132 & U10 & U11 \\
\hline 133 & U10 & MSIQ \\
\hline 134 & U10 & TG2A+0,5TV2C (Diesel) \\
\hline 135 & U11 & MSIQ \\
\hline 136 & U11 & TG2A+0,5TV2C (Diesel) \\
\hline 137 & MSIQ & TG2A+0,5TV2C (Diesel) \\
\hline
\end{tabular}




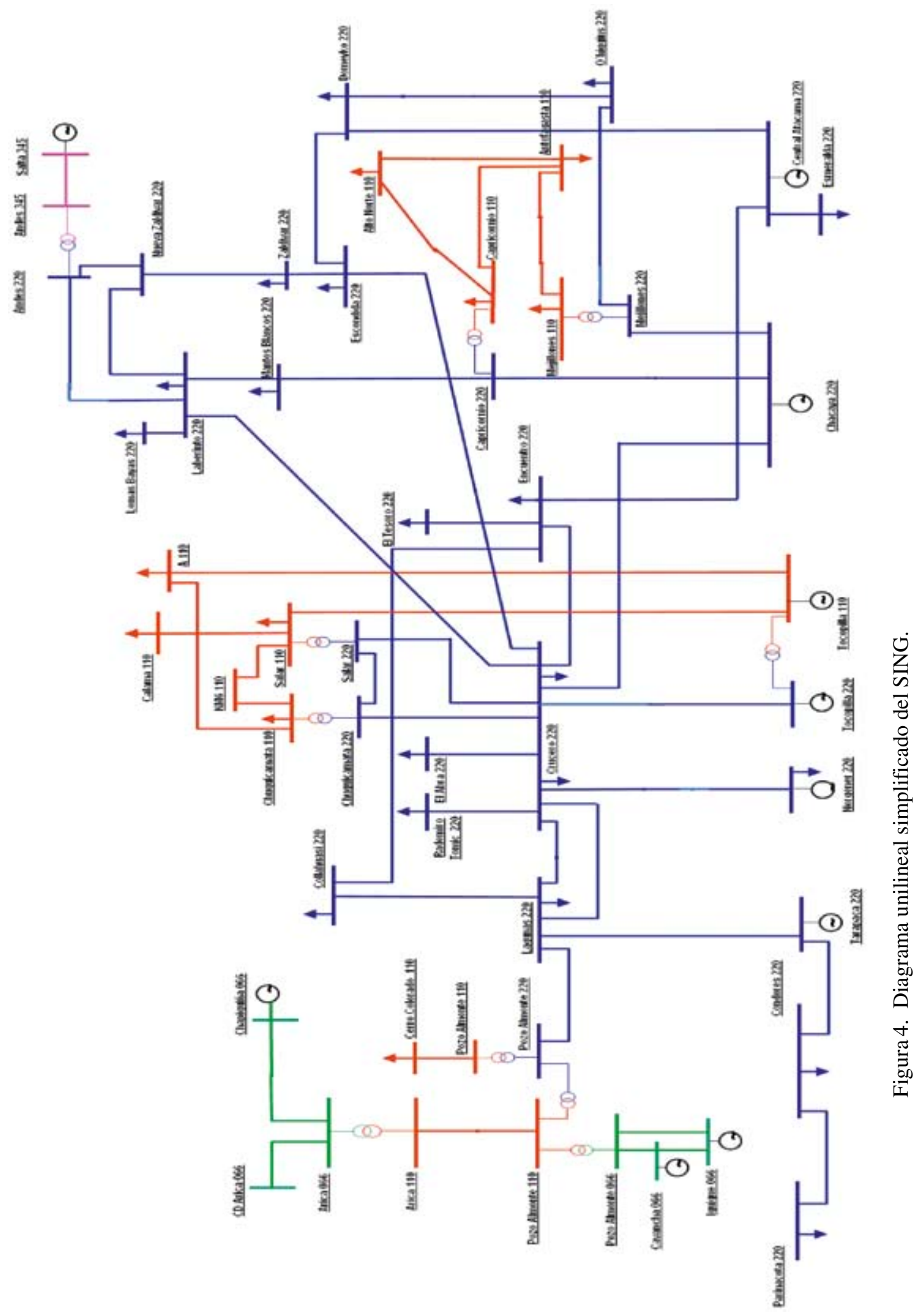


A continuación, las Tablas 2 y 3 resumen el número total de estados seleccionados tanto en demanda máxima como en demanda mínima, respectivamente, para los tres escenarios de análisis considerados. Además, se muestra el porcentaje de estados cubiertos en los tres escenarios y se consideran contingencias de segundo orden como límite para la selección de los estados también en los tres escenarios.

Tabla 2. Número total de estados seleccionados y porcentaje de estados cubiertos en demanda máxima.

\begin{tabular}{|c|c|c|}
\hline \multicolumn{3}{|c|}{ Demanda máxima } \\
\hline Sistema & $\mathbf{N}^{\mathbf{0}}$ Estados & $\begin{array}{c}\text { Porcentaje } \\
\text { estados cubiertos }\end{array}$ \\
\hline GENERACIÓN & 137 & $96,57 \%$ \\
\hline TRANSMISIÓN & 3.241 & $99,90 \%$ \\
\hline COMPUESTO & 4.657 & $93,60 \%$ \\
\hline
\end{tabular}

Tabla 3. Número total de estados seleccionados y porcentaje de estados cubiertos en demanda mínima.

\begin{tabular}{|c|c|c|}
\hline \multicolumn{3}{|c|}{ Demanda mínima } \\
\hline Sistema & $\mathbf{N}^{\mathbf{0}}$ Estados & $\begin{array}{c}\text { Porcentaje } \\
\text { estados cubiertos }\end{array}$ \\
\hline GENERACIÓN & 106 & $97,79 \%$ \\
\hline TRANSMISIÓN & 3.241 & $99,90 \%$ \\
\hline COMPUESTO & 4.466 & $95,30 \%$ \\
\hline
\end{tabular}

El porcentaje de estados cubiertos es un porcentaje que depende, directamente, de las tasas de falla que se utilicen para modelar las fallas de los componentes del sistema. Por lo tanto, al observar los porcentajes de estados cubiertos en las Tablas 2 y 3 y con las tasas de falla estándares para los componentes del sistema [10-11], se puede apreciar que la probabilidad de estados cubiertos se encuentra sobre el $95 \%$, en la mayoría de los casos, considerando contingencias de segundo orden. Este porcentaje de estados cubiertos es bastante aceptable, por lo que no es necesario considerar un orden mayor de contingencias para alcanzar el $100 \%$ de estados cubiertos.

\section{Evaluación de las funciones de prueba}

En esta etapa, corresponde evaluar el comportamiento del sistema frente a los requerimientos de demanda y las contingencias que ocurren durante la operación del mismo. Todos los estados seleccionados de las
Tablas 2 y 3 son analizados para evaluar la función de prueba asociada al índice de confiabilidad que se desea calcular. Para realizar la evaluación de la función de prueba se utiliza la herramienta "Análisis de Contingencias" del programa de flujos de potencia PowerWorld Simulator [12]. Este es un programa muy simple de utilizar, ya que permite presentar, en pantalla, todo el sistema eléctrico de potencia que se desea simular y con el cual se pueden analizar todas las contingencias posibles de ocurrir. Las contingencias que el programa contempla son las siguientes:

- Sacar de servicio las líneas de transmisión del sistema, individualmente.

- Sacar de servicio los transformadores del sistema, individualmente.

- Sacar de servicio las líneas de transmisión o los transformadores, individualmente.

- Sacar de servicio los generadores del sistema, individualmente, $\mathrm{o}$

- Sacar de servicio combinaciones entre líneas de transmisión, generadores y transformadores.

El programa al realizar la simulación define un nuevo despacho acorde a la demanda modelada y muestra las tensiones en cada barra del sistema. Se asume como estado base del sistema, el estado donde no existen componentes fuera de servicio.

Por otro lado, para tener una referencia de los rangos de tensiones permitidos en la operación normal de un sistema eléctrico de potencia en Chile, se recurre a la Norma Técnica de Seguridad y Calidad de Servicio [13], cuyos rangos se detallan en la Tabla 4.

Tabla 4. Rango permitido para las tensiones en las barras del SING.

\begin{tabular}{|c|c|c|c|}
\hline \multirow{2}{*}{$\begin{array}{c}\text { Nivel de } \\
\text { tensión }\end{array}$} & \multicolumn{3}{|c|}{ Rango permitido [p.U.] } \\
\cline { 2 - 4 } & $\begin{array}{c}\text { Estado } \\
\text { normal }\end{array}$ & $\begin{array}{c}\text { Estado } \\
\text { alerta }\end{array}$ & $\begin{array}{c}\text { Estado } \\
\text { emergencia }\end{array}$ \\
\hline $500 \mathrm{kV}$ & {$[0,97 ; 1,03]$} & {$[0,96 ; 1,04]$} & {$[0,95 ; 1,05]$} \\
\hline $345 \mathrm{kV}$ & {$[0,95 ; 1,05]$} & {$[0,93 ; 1,07]$} & {$[0,90 ; 1,1]$} \\
\hline $220 \mathrm{kV}$ & {$[0,95 ; 1,05]$} & {$[0,93 ; 1,07]$} & {$[0,90 ; 1,1]$} \\
\hline $110 \mathrm{kV}$ & {$[0,93 ; 1,07]$} & {$[0,91 ; 1,09]$} & {$[0,90 ; 1,1]$} \\
\hline $66 \mathrm{kV}$ & {$[0,93 ; 1,07]$} & {$[0,91 ; 1,09]$} & {$[0,90 ; 1,1]$} \\
\hline
\end{tabular}

\section{Evaluación del sistema de generación}

Los estados que se consideran como estados de falla en este sistema son los siguientes: 
- Aquellos estados que provocan una insuficiencia de generación para abastecer la demanda del sistema.

- Aquellos estados que dejan la reserva disponible de generación en cero, reserva que corresponde a 216 MW en demanda máxima y 228 MW en demanda mínima.

- Aquellos estados que provocan una variación en las tensiones de barra fuera del rango permitido para un estado de emergencia, según Tabla 4.

\section{Evaluación del sistema de transmisión}

Los estados que se consideran como estados de falla en este sistema son los siguientes:

- Aquellos estados que provocan una pérdida generalizada de carga o blackout.

- Aquellos estados que provocan un desprendimiento de carga producto de sobrecargas en las líneas de transmisión o en los transformadores.

- Aquellos estados que provocan un desprendimiento de carga producto de tensiones fuera del rango permitido para un estado de emergencia según la Tabla 4.

\section{Evaluación del sistema compuesto}

Para la evaluación de este sistema se considera el principio señalado en el punto 2.2, que corresponde a la coherencia del sistema, es decir, el rendimiento del sistema no puede ser mejor si un componente que está funcionando falla, y no puede ser peor si un componente que ha fallado es reparado [6]. Así, los estados considerados de falla en este sistema son los mismos que fueron considerados en los sistemas de generación y transmisión por separados.

\section{Cálculo de los índices de confiabilidad}

En esta etapa se calculan los índices de confiabilidad del sistema mediante las ecuaciones (3), (4), (5) y (6) para cada estado seleccionado y mediante las ecuaciones (7), (8), (9) y (10) para el sistema en general. Las Tablas 5, 6 y 7 entregan los índices de confiabilidad, en ambos niveles de demanda, para los sistemas de generación, transmisión y compuesto, respectivamente, según las tasas de fallas consideradas en [11] y [10]. Cabe agregar que los índices de confiabilidad EPNS y EENS sólo se calcularon para el sistema de transmisión por ser este sistema el de mayor interés en este documento.

\section{Índices desagregados}

Una vez calculados los índices de confiabilidad del sistema de generación, considerando $100 \%$ de disponibilidad del sistema de transmisión, los índices de confiabilidad del sistema de transmisión,

Tabla 5. Índices de confiabilidad del sistema de generación

\begin{tabular}{|c|c|c|}
\hline \multirow{2}{*}{ Sistema de generación } & \multicolumn{2}{|c|}{ Índices de confiabilidad } \\
\cline { 2 - 3 } & LOLP [p.u.] & LOLE [Horas/año] \\
\hline DDA. MÁXIMA & 0,044788354 & 392 \\
\hline DDA. MÍNIMA & 0,065759860 & 576 \\
\hline
\end{tabular}

Tabla 6. Índices de confiabilidad del sistema de transmisión.

\begin{tabular}{|c|c|c|c|c|}
\hline \multirow{2}{*}{$\begin{array}{c}\text { Sistema de } \\
\text { transmisión }\end{array}$} & \multicolumn{4}{|c|}{ Índices de confiabilidad } \\
\cline { 2 - 5 } & LOLP [p.u.] & LOLE [Horas/año] & EPNS [MW] & EENS [MWh/año] \\
\hline DDA. MÁX. & 0,02214968 & 194 & 1,04472044 & 7.962 \\
\hline DDA. MÍN. & 0,019763722 & 173 & 0,658113263 & 5.016 \\
\hline
\end{tabular}

Tabla 7. Índices de confiabilidad del sistema compuesto.

\begin{tabular}{|c|c|c|}
\hline \multirow{2}{*}{ Sistema compuesto } & \multicolumn{2}{|c|}{ Índices de confiabilidad } \\
\cline { 2 - 3 } & LOLP [p.u.] & LOLE [Horas/año] \\
\hline DDA. MÁXIMA & 0,054269456 & 475 \\
\hline DDA. MÍNIMA & 0,072581515 & 636 \\
\hline
\end{tabular}


considerando $100 \%$ de disponibilidad del sistema de generación, y los índices de confiabilidad del sistema compuesto, generación-transmisión, corresponde ahora desagregar el índice compuesto para obtener los reales aportes que cada zona funcional realiza por separado. Para obtener los índices de confiabilidad desagregados $\overline{\mathrm{PENS}}_{\mathrm{T}}$ y $\overline{\mathrm{EENS}}_{\mathrm{T}}$ del sistema de transmisión, se utiliza un factor multiplicativo que proviene del cociente entre el índice desagregado $\overline{\mathrm{LOLP}}_{\mathrm{T}}$ y el índice preliminar LOLP $\mathrm{T}$. Por lo tanto, los índices de confiabilidad desagregados para los sistemas de generación y transmisión del SING se muestran en las Tablas 8 y 9 .

Luego de calcular los índices de confiabilidad desagregados para los sistemas de generación y transmisión, se analiza, económicamente, el comportamiento del sistema de transmisión frente a las indisponibilidades que pueden ocurrir, y determinar el factor de sobrecosto asociado, el cual se analiza en el siguiente punto.

Tabla 8. Índices desagregados en demanda máxima.

\begin{tabular}{|c|c|c|}
\hline \multicolumn{3}{|c|}{ Demanda máxima } \\
\hline Índices & Transmisión & Generación \\
\hline$\overline{L O L P}$ [p.u.] & 0,017957669 & 0,036311786 \\
\hline$\overline{L O L E}[$ Horas/año] & 157 & 318 \\
\hline$\overline{E P N S}[\mathrm{MW}]$ & 0,84699866 & - \\
\hline$\overline{E E N S}[\mathrm{MWh} / \mathrm{año}]$ & 6.455 & - \\
\hline
\end{tabular}

Tabla 9. Índices desagregados en demanda mínima.

\begin{tabular}{|c|c|c|}
\hline \multicolumn{3}{|c|}{ Demanda mínima } \\
\hline Índices & Transmisión & Generación \\
\hline$\overline{L O L P}$ [p.u.] & 0,016772928 & 0,055808586 \\
\hline$\overline{L O L E}[$ Horas/año] & 147 & 489 \\
\hline$\overline{E P N S}[\mathrm{MW}]$ & 0,558522641 & - \\
\hline$\overline{E E N S}[\mathrm{MWh} / \mathrm{año}]$ & 4.257 & - \\
\hline
\end{tabular}

\section{CÁLCULO DEL FACTOR DE SOBRECOSTO ASOCIADO A LA INDISPONIBILIDAD DEL SISTEMA DE TRANSMISIÓN}

Parte importante del objetivo central de este trabajo es calcular el factor de sobrecosto asociado a la indisponibilidad del sistema de transmisión del SING, de manera de conocer el comportamiento económico del sistema frente a los distintos niveles de demanda y frente a las condiciones naturales de los componentes del sistema cuando fallan. En este trabajo se utilizan las fórmulas desarrolladas en [14] para calcular el factor de sobrecosto asociado a un sistema eléctrico de potencia. En [14] se afirma que cuando un sistema se encuentra operando con un cierto nivel de demanda existe un costo marginal asociado a dicho nivel de operación que, generalmente, está relacionado con la central térmica despachada que posee mayor costo variable de operación. Si se supone que tal afirmación es correcta, cumpliéndose para el SING y que, además, el costo en que incurre el sistema cuando se produce una falla es el costo que se ha denominado en la ley chilena como costo de falla de corto plazo, entonces es posible utilizar las fórmulas de [14] para calcular el factor de sobrecosto asociado a la indisponibilidad del sistema de transmisión. Dichas ecuaciones se presentan a continuación.

$$
\begin{gathered}
\mathrm{CMg}_{\text {equivalente }}=\mathrm{CMg}_{\text {base }} \cdot\left(1-\overline{\mathrm{LOLP}_{\mathrm{T}}}\right)+\mathrm{CMg}_{\text {falla }} \cdot \overline{\mathrm{LOLP}_{\mathrm{T}}} \\
\text { Factor de sobrecosto }=\frac{\mathrm{CMg}_{\text {equivalente }}}{\mathrm{CMg}_{\text {base }}}
\end{gathered}
$$

Donde:

$\mathrm{CMG}_{\text {equivante }}$ : Costo marginal equivalente del sistema en operación normal y bajo un cierto nivel de demanda.

$\mathrm{CMG}_{\text {base }} \quad$ : Costo marginal base del sistema, en operación normal y bajo un cierto nivel de demanda.

$\mathrm{CMG}_{\text {falla }}$ : Costo marginal de falla del sistema, declarado semestralmente en el informe técnico preliminar de precios de nudo.

El costo marginal equivalente mostrado en la ecuación (19), al ser dividido por el costo marginal base, se obtiene el factor de sobrecosto que surge producto de las indisponibilidades del sistema de transmisión. Este factor de sobrecosto se utiliza para reflejar la confiabilidad del sistema de transmisión, desde la perspectiva de la capacidad o suficiencia, en los precios de nudo. Con respecto al costo de falla utilizado, corresponde al costo de falla declarado en el informe técnico preliminar de precios de nudo del SING de abril de 2007, cuyo 
valor es 335 [U\$S/MWh] [15]. El costo marginal base del SING es determinado en base al despacho imperante en el sistema, donde la unidad que posee mayor costo variable, dentro de la lista de méritos, es la que marca el costo marginal de operación. Así, en demanda máxima para este estudio, la unidad que margina según el despacho utilizado para el SING es la unidad de ciclo combinado $\mathrm{N}^{\circ} 1$ diesel (CC1-diesel) de la Central Atacama, cuyo costo marginal es $\lambda=88,463[\$ / \mathrm{kWh}]$, mientras que para la demanda mínima la unidad que margina es la unidad $\mathrm{N}^{\mathrm{o}} 11$ (U11) de la Central Termoeléctrica Tocopilla, cuyo costo marginal es $\lambda=85,06[\$ / \mathrm{kWh}]$ [16]. La Tabla 10 entrega los costos marginales base y equivalente, tanto para la demanda máxima como para la demanda mínima del SING.

Tabla 10. Costos marginales del SING.

\begin{tabular}{|c|c|c|}
\hline Costos marginales & DDA. máxima & $\begin{array}{c}\text { DDA. } \\
\text { mínima }\end{array}$ \\
\hline $\mathrm{CMG}_{\text {base }}[\mathrm{US} \$ \mathrm{MWh}]$ & 163,14 & 156,76 \\
\hline $\mathrm{CMG}_{\text {equivalente }}[\mathrm{US} \$ / \mathrm{MWh}]$ & 166,23 & 159,75 \\
\hline
\end{tabular}

Luego, con ambos costos marginales equivalentes calculados, se determina el factor de sobrecosto asociado a la indisponibilidad del sistema de transmisión, mostrados en la Tabla 11.

Tabla 11. Factor de sobrecosto en ambos niveles de demanda.

\begin{tabular}{|c|c|c|}
\hline & DDA. máxima & DDA. mínima \\
\hline $\begin{array}{c}\text { Factor de } \\
\text { sobrecosto [p.u.] }\end{array}$ & 1,018917525 & 1,019071234 \\
\hline
\end{tabular}

Los factores entregados en la Tabla 11 fueron calculados con los costos base y equivalente entregados en la Tabla 10.

\section{ANÁLISIS, CONCLUSIONES Y TRABAJO FUTURO}

Una característica importante de la evaluación de la confiabilidad por medio del método utilizado es considerar un número reducido de estados que forman, en definitiva, un subconjunto de estados del conjunto total de estados posibles. Esta característica responde a las mejoras que se le han incorporado al método, con el afán de disminuir los tiempos de ejecución de la herramienta, sin perder precisión ni generalidad en los resultados. La idea de trabajar con subconjuntos de estados es simplificar la cantidad de estados a analizar. En rigor, evaluar la confiabilidad del SING significaba analizar 65.536 estados para el sistema de generación, 1,2 $\mathrm{e}^{24}$ estados para el sistema de transmisión y $1,6 \mathrm{e}^{29}$ estados para el sistema compuesto, pero, según las Tablas 2 y 3 , la cantidad de estados seleccionados y analizados es bastante menor, con porcentajes de ocurrencia sobre el $95 \%$, que es bastante aceptable para obtener índices de confiabilidad representativos del sistema. Estos subconjuntos de estados suelen formarse considerando un cierto nivel de contingencias que dependen del sistema en estudio y del nivel de detalle que se necesite para su análisis, pero por lo general, considerar hasta un nivel de contingencia de segundo orden, se cubre un alto porcentaje de ocurrencia de los estados. Existen ciertas excepciones a esto último, excepciones que están ligadas con las tasas de falla y reparación que se utilicen para modelar las fallas de los componentes del sistema, ya que si los componentes tienen un porcentaje de disponibilidad, que en promedio supera el $90 \%$, considerar hasta un nivel de contingencias de segundo orden se cubre sobre el $90 \%$ de las probabilidades de ocurrencia de los estados, pero si el porcentaje de disponibilidad, en promedio, baja del $90 \%$, es necesario seleccionar niveles de contingencias mayores a segundo orden para cubrir sobre el $90 \%$ de ocurrencia. Lo mismo ocurre con la disponibilidad de los tramos de línea, dado que si el porcentaje de disponibilidad, en promedio, bordea el $99 \%$, considerar contingencias de hasta primer orden ya se estarían cubriendo casi el $100 \%$ de los estados, pero si el porcentaje de disponibilidad promedio baja del $95 \%$, se deben considerar niveles de contingencias superiores a segundo orden, para cubrir, por lo menos, el $90 \%$ de ocurrencia de los estados.

Con respecto al factor de sobrecosto asociado a la indisponibilidad del sistema de transmisión del SING, se puede decir que es una variable muy importante dado que permite reflejar la confiabilidad del sistema de transmisión en el proceso de fijación de precios de nudo calculado por la CNE. Dicho ente regulador declara que el factor de sobrecosto asociado a la indisponibilidad del sistema de trasmisión es de 1,000085 p.u., sobrecosto bastante bajo para el tipo de sistema que es el SING, y que además, según las observaciones a los informes técnicos de precios de nudo [15], no estaría reflejando los reales sobrecostos incurridos para mantener la suficiencia del sistema. 
Es así como en este trabajo se demuestra que el índice de indisponibilidad y el factor de sobrecosto asociado son mayores bajo consideraciones de simulación más reales a la operación normal del sistema. Por otro lado, el índice de confiabilidad desagregado $\overline{\mathrm{LOLE}}_{\mathrm{T}}$, obtenido tanto para la demanda máxima como para la mínima, es el índice de confiabilidad más importante de este documento, ya que cuantifica la cantidad de horas al año en que este sistema no podrá abastecer la demanda total existente. Dicho índice, al ser contrastado con el declarado por la CNE en los procesos de fijación de precios de nudo [17], especificando que la indisponibilidad de transmisión del SING es de 0,24 horas/año, se puede notar la gran diferencia existente, producto de que la CNE considera algunos aspectos muy cuestionados por las empresas eléctricas [15]. Pues bien, estas diferencias se explican en el contexto de los distintos aspectos considerados para modelar al sistema. Entre estos aspectos se pueden mencionar los siguientes:

1. La salida de servicio de todos los tramos de línea que el modelo utilizado del SING contempla, bajo contingencias de primer orden y segundo orden. La CNE declara sólo sacar de servicio 23 tramos de línea, desconociendo cuáles son y el por qué de dicha condición.

2. El índice calculado proviene de la desagregación del índice compuesto, es decir, no se está subestimando el efecto que tienen las indisponibilidades de las unidades generadoras cuando ocurren fallas en los tramos de línea del sistema.

3. Finalmente, el índice de indisponibilidad se obtiene con estadísticas de falla estándares, no siendo el caso de la CNE que considera estadísticas de falla no estándares y muy cuestionadas en las observaciones de los informes técnicos preliminares de precios de nudo.

Ahora bien, al haber calculado el factor de sobrecosto asociado a la indisponibilidad de transmisión del SING, para dos niveles de demanda, fue posible conocer el comportamiento de este factor en otro escenario que no fuese la demanda punta anual típica que se considera en los estudios de los sistemas eléctricos de potencia, que suele ser un criterio fuerte de análisis. Este factor en demanda mínima no presenta diferencias significativas con respecto al factor de sobrecosto que surge en demanda máxima, situación que, quizás, justifica al ente regulador, el que calcula este factor sólo para la demanda máxima del sistema.

Con respecto a las consideraciones realizadas en la aplicación de la metodología, se destaca que entrega resultados aceptables, basados en dos razones: i) la metodología no está sujeta a grandes errores, y ii) los cálculos realizados son relativamente simples, pero laboriosos. Cabe agregar, además, que una de las variables de entrada más importantes en el cálculo de los índices de confiabilidad, es la tasa de falla que determina la indisponibilidad de los componentes del sistema [10]. En Chile, una de las observaciones más frecuentes realizadas por las empresas del SING, en los procesos de fijación de precios de nudo, es el origen que tiene la tasa de falla para las líneas de transmisión, equivalente a 0,000176 horas $/ \mathrm{km}$ al año. Según lo analizado a lo largo de este estudio, los índices de confiabilidad están directamente relacionados con las tasas de falla, siendo indispensable tener definido qué tasas de falla utilizar antes de evaluar la confiabilidad de un sistema. Es por esto que, en este trabajo, se calcularon los índices de confiabilidad, con tasas de fallas estándares, de manera de evitar cuestionamientos acerca de la procedencia de estas mismas. Los índices de confiabilidad calculados en este trabajo corresponden a valores esperados, valores que se cumplen en la práctica, en la medida que los eventos de falla de los componentes del sistema sean similares a las tasas de fallas estándares utilizadas.

Como desarrollos futuros, se propone complementar esta metodología probabilística con la incorporación de mayor información que permita calcular, además de los índices ya obtenidos en este trabajo, otros índices de confiabilidad que den cuenta, por ejemplo, de la frecuencia con que ocurre una contingencia o falla, y la duración media de dicha falla, por mencionar algunos, para entender y evaluar de mejor manera el comportamiento del sistema.

También se propone como desarrollos futuros realizar una evaluación de la confiabilidad del SING, mediante una metodología basada en la simulación de Monte Carlo, que corresponde al segundo grupo de métodos probabilísticos existentes para evaluar la confiabilidad de los sistemas eléctricos de potencia. De esta manera, el espectro de índices 
que se puede obtener para el SING será tal, que se podrá contar con una imagen casi real de la operación del sistema, en cuanto a su confiabilidad. Cabe mencionar que una metodología basada en la simulación implica, muchas veces, niveles altos de complejidad, tanto en términos computacionales como en tiempos de dedicación para la ejecución de la herramienta, ya que se fundamentan en conceptos de programación y avanzada práctica con programas computacionales.

\section{REFERENCIAS}

[1] P. Díaz. "Evaluación de la Confiabilidad en el Marco Reestructurado de los Sistemas Eléctricos Competitivos". Memoria para optar al Título de Ingeniero Civil de Industrias. Pontificia Universidad Católica de Chile. Santiago, Chile. 2000.

[2] M. Alvarez, A. Wilson y E. Coppes. "Análisis de Herramientas para el Estudio de la Confiabilidad de un Sistema Eléctrico de Potencia”. Memoria para optar al Título de Ingeniero Electricista. Universidad de la República Oriental del Uruguay. Montevideo, Uruguay. 2002.

[3] L. Gacés y O. Gómez. "Análisis de Confiabilidad del Sistema de Transporte Regional usando Simulación de Monte Carlo". Tesis de grado para optar al título de Ingeniero Electricista. Universidad Tecnológica de Pereira. Pereira, Colombia. 2003.

[4] Software estadístico "StatGraphics" versión 5.1. Edición profesional. Copyright 1994-2000 Statistical Graphics Corp.

[5] A. Gómez Expósito. "Análisis y Operación de Sistemas de Energía Eléctrica". McGraw-Hill. España. 2002.

[6] L.R. Larraín. "Cálculo de un Índice de Indisponibilidad de Transporte para el Sistema Interconectado Central basado en el Método Predictivo Probabilístico Analítico". Memoria para optar al Título de Ingeniero Civil de Industria con diploma en Ingeniería Eléctrica. Pontificia Universidad Católica de Chile. Santiago, Chile. 2001.

[7] S. Oren. "Ensuring Generation Adequacy in Competitive Electricity Markets". University of California at Berkeley. Prepared under contract from the Electric Power Research Institute. USA. 2004.
[8] R. Billinton and R. Allan. "Reliability Evaluation of Engineering Systems: Concepts and Techniques". Plenum Press. New Cork, NY, USA. 1983.

[9] CDEC-SING-2007. "Antecedentes y Parámetros Técnicos de las Instalaciones del SING. 20062007". Fecha de consulta: 23 de noviembre del 2007. URL: http://www.cdec-sing.cl

[10] "Generating Availability Data System". (GADS) Reporting 2006, NERC. Date of visit: 10 de Octubre del 2007. URL: http://www.nerc.com

[11] CDEC-SING-2007. Actividades Norma Técnica. Reporte de Estudios. Estudio No 9 (DP): "Estudio de Continuidad". 2007. Fecha de consulta: 4 de diciembre del 2007. URL: http://www.cdec-sing.cl.

[12] Software de Flujos de Potencia "PowerWorld Simulator" version 8.0 OPF, ATC, PVQV. 2004 South Wright St. Urbana, IL 61801. Licencia válida sólo para facultades, estudiantes y staff universitarios. Disponible en el Departamento de Ingeniería Eléctrica. Facultad de Ingeniería. Universidad de Antofagasta. Chile.

[13] COMISIÓN NACIONAL DE ENERGÍA (CNE). "Norma Técnica de Seguridad y Calidad de Servicio". Santiago de Chile. 2005. Fecha de consulta: 14 de abril del 2007.

URL: http://www.cdec-sing.cl

[14] J.I. Vergara Correa. "Aspectos Técnicos y Remunerativos de los Servicios Complementarios en Sistemas Eléctricos Desregulados". Memoria para optar al Título Ingeniero Civil de Industrias. Pontificia Universidad Católica de Chile. Santiago, Chile. 2000.

[15] COMISIÓN NACIONAL DE ENERGÍA (CNE). "Fijación de precios de nudo. Abril de 2007". Sistema Interconectado del Norte Grande (SING). Informe Técnico Preliminar. Fecha de consulta: 3 de mayo del 2007. URL: http://www.cdec-sing.cl

[16] CDEC-SING-2007. "Planificación y Programación. Lista de Méritos de las Unidades Generadoras (TCV)". Fecha de consulta: 23 de noviembre del 2007.

URL: http://www.cdec-sing.cl

[17] COMISIÓN NACIONAL DE ENERGÍA (CNE). "Observaciones al Informe Técnico Preliminar (ITP) de fijación de precios de nudo. Abril de 2007". Sistema Interconectado del Norte Grande (SING). Fecha de consulta: mayo del 2007. URL: http://www.cdec-sing.cl 